\title{
THE EFFECT OF TEACHING THE PRINCIPLES OF BEHAVIOR MANAGEMENT ON PARENT-CHILD RELATIONSHIP PARENTS OF CHILDREN WITH ADHD
}

\author{
Mahdieh GHALENOEE ${ }^{1}$, Zahra MADDAH ${ }^{2 *}$, Jamileh MOHTASHAMI ${ }^{3}$, Mohamad Amin \\ POURHOSEINGHOLI ${ }^{4}$, Roghayeh ESMAIELI ${ }^{5}$
}

\begin{abstract}
${ }^{1}$ MSc, Psychiatric Nursing Department, School of Nursing and Midwifery, Shahid Beheshti University of Medical Science, Tehran, Iran.

${ }^{2}$ MSc, Psychiatric Nursing, School of Nursing and Midwifery, Saveh University of Medical Sciences, Saveh, Iran.

${ }^{3}$ Assistant Professor, Psychiatric Nursing Department, School of Nursing and Midwifery, Shahid Beheshti University of Medical Sciences, Tehran, Iran.

${ }^{4}$ Assistant Professor of Biostatistics Department of Health System Research, Research Institute for Gastroenterology and Liver Diseases, Shahid Beheshti University of Medical Sciences, Tehran, Iran.

${ }^{5}$ Assistant Professor in Nursing Field, School of Nursing and Midwifery, Shahid Beheshti University of Medical Science, Tehran, Iran.
\end{abstract}

${ }^{*}$ Corresponding Author

\section{ABSTRACT}

Introduction \& O6jective: Parental management training is a necessary part of mental healt $\mathrm{interventions} \mathrm{for} \mathrm{attention-}$ deficit / hyperactivity disorder. But the results from past studies provide controversial information on the impact of parent behavior management training programs on reducing the atypical behaviors of children with attention deficit and hyperactivity disorder (ADHDD), so this study aimed to determine the effect of parent education curriculum on the parentchild relationship, parents of children with ADHDD. Method: This study was an experimental study in which two intervention and control groups were compared. In this research, two questionnaires were used: the first questionnaire was related to demographic information and the second questionnaire was related to Parker, Tupling, and Brown. In the end, all statistical analyses were done with the statistical package of social science software version 21. Results: The results of this study showed that parenting education program has a positive effect on parent/child relationship, parents of children with attention-deficit / hyperactivity disorder and is significant on $\mathbb{P} \leq 0.05$. The affected child is promoted by the intervention. Conclusion: Parent management education is of paramount importance for children with ADHD and can have a positive effect on the parent-child relationship and increase the quality of life in families of these children.

Keywords: Gehavior management, parent-child relationship, attention deficit disorder/hyperactivity disorder, parental education.

\section{INTRODUCTION}

Currently, the diagnosis of ADHD is based on the consensus opinion of the experts who believe that all three visible types of disorder, namely, neglect, hyperactivity/impulsivity, or composite are manifestations of this disorder. Concerning the etiology of this disorder, there is currently a consensus that the cause of attention deficit hyperactivity disorder (ADHD) is the complex interaction of neuromuscular and neurochemical systems. This opinion consensus is based on family junctures, twins and adolescents, studies on dopamine vector gene, neural imaging, and neurotransmitter data (Pelham and Hoza, 2010). Factors that are considered for attention deficit/hyperactivity disorder include: exposure to toxins in preterm birth, early pregnancy, and prenatal mechanical damage to the fetal nervous system, food additives, colors, preservatives, 
and sugars also the title of the probable causative agent of overactive behavior has been raised. But scientific evidence does not indicate the involvement of these factors in the onset of ADHD (Hetchman et al, 2009).

Treatment of a child with ADHD includes different dimensions: family education and counseling, drug therapy, appropriate classroom selection, environmental changes, and social and psychological interventions. The results of studies have shown that drug use alone can often not meet the comprehensive needs of children with this disorder, and only one aspect of the multi faceted program is the treatment of this disorder (Rajablo, 2012). Parent management education is a necessary part of mental health interventions for attention deficit/hyperactivity disorder. The basis of most parenting education is helping to create useful behavioral interventions with positive reinforcement targeting both academic behaviors and social behaviors. Group therapy can be very useful for improving children's social skills and self esteem and the sense of success in children suffering from a group of children, especially at school (Sadok and Sadok, 2014). Parent management education programs are based on the principles of social learning and teaching parents how to establish a more positive interaction with children and reduce the level of negative behaviors and internal conflicts in the family. Results from Ostberg and Rydell's studies in 2012 showed that parenting education would reduce the diagnostic criteria for attention - deficit/hyperactivity disorder in children. However, the results of Enebrink et al. (2012) and Barkley et al. (2008) showed that the parental education management program was not effective in reducing hyperactivity Signs and Symptoms. Considering the controversial results about the effect of the parent behavior management training program on reducing the atypical behaviors of children with ADHD, as well as considering that so far, none of the studies have individually and specifically influenced the program. This study was conducted to determine the effect of parent management education programs on parent/child relationships between parents and their children with ADHD.

\section{METHOD}

This study was an experimental study in which two groups of intervention and control were compared to determine the effect of parent education program based on the parental management education model on the parent/child relationship between parents and their children with ADHD. In this study, 40 parents (father or mother) of children with ADHD in Saveh city who had the criteria for entering the study and were satisfied to participate in this study. The criteria for entry into this study included: willingness to participate in the study, at least 6 months were passed from the diagnosis and treating the childhood disorder, lack of physical disorder, mental disorder, mental retardation, and chronic diseases in the child with attention $\sim$ deficit/Hyperactivity, at least reading and writing literacy in parents, the absence of significant physical and mental disorders in the parents of childhood ADHD and the lack of parental involvement in the same educational programs in the last 6 months and exclusion criteria including non participation in 2 of the meetings were attended by training sessions and attending similar sessions or training programs simultaneously.

In this research, a two stage sampling method was used in which the first stage was based on purpose sampling and the second stage was the random allocation method. 
The minimum number of samples was obtained using the following equation in each group with 20 people:

$\mathrm{n}=\left(\left(\mathrm{z}_{-}(\mathrm{a} / 2)+\mathrm{z}_{-} \beta\right) \wedge 2 \sigma^{\wedge} 2\right) / \varepsilon^{\wedge} 2$

Wherein:

The probability of a type 1 error: $\alpha=0.05 \rightarrow$ z_ $\alpha=1.96$

The power of the test is $1 \sim \beta=0.8$

The rate of change in the relationship index (according to Khoshabi et al. (2013) after the intervention was $\varepsilon=2$ and the standard deviation from this study was considered as 4.5. The sample size required was 40 , which was used to prevent possible dropping of samples, $15 \%$ was added to the samples, totaling 44 people (40 parents with children with ADHD were included in the study).

Two questionnaires were used in this study. The first questionnaire was related to demographic information, which consisted of two parts of the questionnaire. The first part consisted of 4 questions about the child (age of the child, child sex, duration of attention deficit/hyperactivity disorder diagnosis, and specific physical illness in the child) and the second part consisted of 8 questions about the parent (father's age, mother's age, father's level of education, Mother's level of education, father's occupation, mother's occupation, number of children with ADHD, history of parental involvement in psychiatric disorders, history of use of psychiatric drugs in parents). The second part of the questions also included the Family Linkage Questionnaire, which was made by Parker, Tupling, and Brown in 1979. In the case of validity, the Family Relationship Questionnaire was well documented and had a significant correlation with independent judgmental and parental attention and support. On the whole, the attention and support of mothers were more meaningful than the attention and support of fathers. To determine the reliability of the questionnaire, the internal consistency method was used to calculate the Cronbach's alpha coefficient and the re test method. In this study, Cronbach's alpha coefficient was 0.76. In this study, an educational program based on the Barclay model (1997) was used to implement the intervention. In this intervention program, 60 minute sessions (twice a week) the week was held in coordination with parents and state counseling authorities. In the first stage (before the start of the training program), responding to Parker's demographic and family history questionnaire and in the second stage (immediately after the last training session) and the third stage (one month after the last session) responding to Parker Family Inventory Questionnaire were conducted by parents.

For data analysis, frequency, mean, standard deviation, and percentages were used to describe the data. By Kolmogorov $\sim$ Smirnov test, the quantitative values were evaluated and the Chi square test was used to determine whether the intervention was effective or not. Covariance analysis in the repeated measurement model was used to control the confounders. All statistical analyses were performed with the statistical package of social science software version 21.

\section{RESULTS}


The results of this study showed that in the control and intervention groups, about $5 \%$ of the participants were children aged 12 years. Also, in the control group, 6, 7 and 11 year old children had the highest (20\%) frequency and in the intervention group, the children $(8,9$, and 10 years old) had the highest (20\%) frequency of the participants In the control group, boys with attention deficit / hyperactivity disorder had (60\%) frequency and the girl children with attention deficit / hyperactivity disorder had (40\%) frequency and in the intervention group boys with attention deficit hyperactivity disorder. / Hyperactivity had (65\%) frequency and girls with attention - deficit / hyperactivity disorder had (35\%) frequency. Also, in the intervention group, the highest number (10 persons) was 12 18 months and the lowest number (1 person) was 2 years old. In the intervention group, in the highest number of patients (8) 12 6 months and the lowest number of patients (2) 24 30 months were passed from the diagnosis time. In the control group, $20 \%$ of children with attention -deficit/hyperactivity disorder had a physical illness and $80 \%$ had no disease. In the intervention group, $15 \%$ of the children had a physical illness and $85 \%$ of the children had no illness. The probability level for the association of specific disease and the frequency of ADHD with the chi square test was more than 0.05, which indicated the lack of correlation between a specific disease and ADHD frequency. The mean and standard deviation of fathers' age in the control and intervention groups were $41 \pm 6.325$ and $41.95 \pm 5.626$, respectively, and the mean and standard deviation of the mothers' age in the control and intervention groups were $36.10 \pm 4.364$ and $30 \pm 4.921 / 37$, respectively. In the case of parental education in the control group, $10 \%$ of children with ADHD had fathers with the least education and $40 \%$ of the children's fathers had high school education, and in the intervention group 5 percent of fathers had elementary education, with the lowest frequency, and 50 percent of fathers had high school education with the highest frequency. The probability of the relationship between father's education and the frequency of ADHD was more than 0.05 using the Chi $\sim$ square test, which indicated that there is no relationship between the parent's education and ADHD frequency. In the control group, $10 \%$ of children with attention deficit/hyperactivity disorder had mothers with the lowest education in the control group, and $35 \%$ of mothers had a high school education and in the intervention group 10\% of the children with ADHD, had mothers with the lowest primary education, and $40 \%$ of mothers had high school education that was most prevalent. The probability level for the relationship between mother's education and the ADHD frequency was more than 0.05 using the Chi rquare test, which indicated that there is no relationship between mother's education and the frequency of ADHD.

But regarding the history of drug use and its relationship with ADHD, the data showed that in the control group, $35 \%$ of the parents had a history of taking psychosocial drugs and $65 \%$ of the parents had no history of taking medication. In the intervention group, $5 \%$ of the parents had a history of consumption of psychiatric drugs and $95 \%$ of the parents had no history of taking medication. The probability of association between the history of drug use and the frequency of ADHD with the chi square test was less than 0.05, which indicated a relationship between the history of drug use and the frequency of ADHD.

Kolmogorov Smirnov test was used to evaluate the normality of data. The results of this study showed that $\mathrm{z} \sim$ Kolmogorov $\sim$ Smirnov statistic for the pre test of care and extreme support were 0.13 and 0.168 , respectively, immediately after completing the exercise education, care and support were 0.125 and 0.130 , and one month after education, care and extreme support were 
0.13 and 0.129 respectively $(\mathrm{P} \leq 0.05)$ that was the distribution of variables in a sample with its distribution in a normal society. Therefore, the assumption of the normalization of data in the community was confirmed.

Regarding the mean and standard deviation of the care and support extreme indexes in the control group, the mean and standard deviation of care scores in the pretest were 18.55 and 6.629 , respectively and the mean and standard deviation of extreme support in the pre-training period were 24.00 and 5.07 , respectively. The mean and standard deviation of care scores immediately after training were 18.65 and 52.33, respectively, and the mean and standard deviation scores of extreme support immediately after training were 24.10 and 525.2, respectively. The mean and standard deviation of care scores a month after training were 18.78 and 6.404, respectively, and the mean and standard deviation scores of extreme support one month after training were 23.83 and 5,227.5, respectively, and in the intervention group, The mean and standard deviation of care scores before training were 16.75 and 5.488, respectively, the mean and standard deviation scores of extreme support before training were 40.33 and 4.286 , respectively, the mean and standard deviation of care scores immediately after training were 22.12 and 5 212.5, the mean and standard deviation scores of extreme support immediately after training were 89.2 and 4.497 , respectively. The mean average and standard deviation of care scores one month after training were 72.26 and 4/599, respectively, and the average score and standard deviation of extreme support one month after training were 20.67 and 22.23 , respectively.

For the present research hypothesis, "Parental management education has an effect on the parent/offspring of children with attention -deficit/hyperactivity disorder one month after training" the results of the Lone test showed that the value of the F test, except for the third time, was not significant and this indicates that the assumption of the equality of variances in extreme protection is the same.

The analysis of repeated measures to compare the mean of care index in two groups with two replications (second and third times)

The results of the internal effects test showed that, since the P values were less than 0.05 , the intervention on the care index was effective over time (Table 1).

Table 1. Interpersonal effects test

\begin{tabular}{|c|c|c|c|c|}
\hline Source of changes & sum of squares & Degrees of freedom & F & P value \\
\hline Time & $78 / 656$ & 1 & $17 / 209$ & $0 / 001<p$ \\
\hline Time* ${ }^{*}$ Group & $87 / 113$ & 1 & $19 / 059$ & $0 / 001<p$ \\
\hline Error (time) & $150 / 830$ & 33 & & \\
\hline
\end{tabular}

Also, the results of the test of the effects among individuals showed that, given that the $\mathrm{P} \sim$ values were less than 0.05, the care index of the response rates of the questionnaires varies in the two intervention and control groups, which means that the intervention was effective. (Table 2). 
Table 2. Between individuals' effects test

\begin{tabular}{|c|c|c|c|c|}
\hline Source of changes & sum of squares & Degrees of freedom & $\mathbf{F}$ & P-value \\
\hline Intercept & $32968 / 136$ & 1 & $537 / 196$ & $\mathrm{p}<0 / 001$ \\
\hline Group & $579 / 108$ & 1 & $9 / 436$ & $0 / 004$ \\
\hline Error (time) & $2025 / 235$ & 33 & & \\
\hline
\end{tabular}

Chart of the values and changes in the mean of care index three times showed that the level of care index in the control group did not change in three periods (before the intervention, immediately after the intervention, and one month after intervention) and showed a constant line chart. However, the index of care in the intervention group increased at the third time (one month after the intervention) compared to the first time (before intervention), which indicated the effectiveness of the parental management training program on the care index (Figure 1).

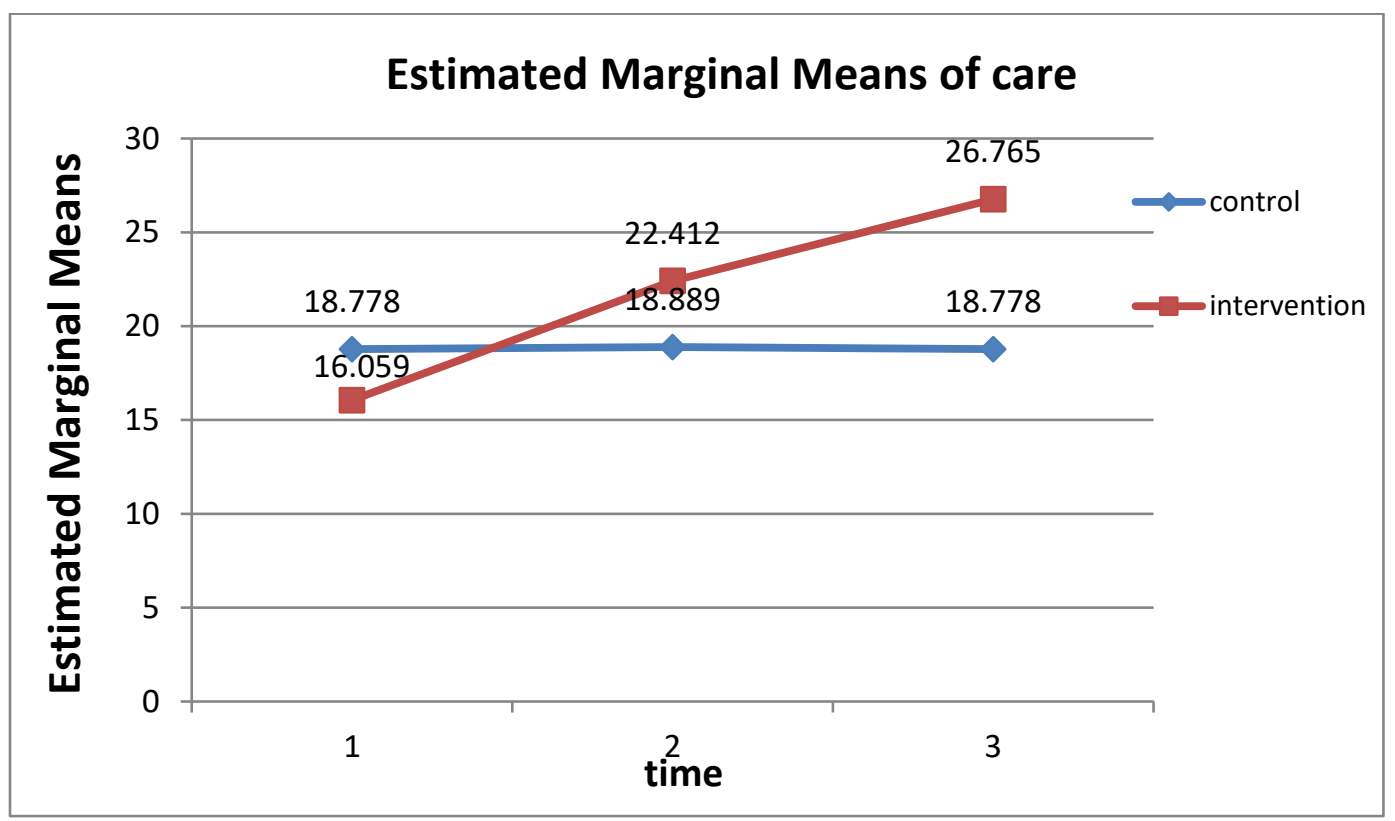

Figure 1. Analysis of the repeated measures to compare the mean of extreme support index in two groups and with two replications (second and third times)

The results of the internal effects test showed that, given that the P values were less than 0.05, the intervention on the extreme support index was effective over time (Table 3).

Table 3. Interpersonal effects test

\begin{tabular}{|c|c|c|c|c|}
\hline Source of changes & sum of squares & Degrees of freedom & F & P -value \\
\hline Time & $2 / 138$ & 1 & $0 / 501$ & $0 / 484$ \\
\hline Time*Group & $0 / 995$ & 1 & $0 / 233$ & $0 / 633$ \\
\hline Error (time) & $150 / 948$ & 33 & & \\
\hline
\end{tabular}


The test of the effects among individuals also showed that in the case of the extreme support index, the response rates of the questionnaires were different in the intervention and control groups, which means that the intervention was effective $(\mathrm{P} \sim$ value $=0.0)($ Table 4$)$.

Table 4. Between individuals' effects test

\begin{tabular}{|c|c|c|c|c|}
\hline Source of changes & sum of squares & Degrees of freedom & F & P value \\
\hline Intercept & $35326 / 334$ & 1 & $1043 / 252$ & $p<0 / 001$ \\
\hline Group & $140 / 048$ & 1 & $4 / 136$ & $0 / 047$ \\
\hline Error (time) & $1117 / 438$ & 33 & & \\
\hline
\end{tabular}

The chart of values and changes in the average of the extreme support index in three times also showed that the value of the extreme support index in the control group did not significantly change during the three periods (before the intervention, immediately after the intervention, and one month after intervention) and almost line diagrams. However, the value of the extreme support index in the intervention group was reduced at the third time (one month after the intervention) compared to the first time (before the intervention), which indicated the effectiveness of the parental management training program on the extreme support index (Figure 2).

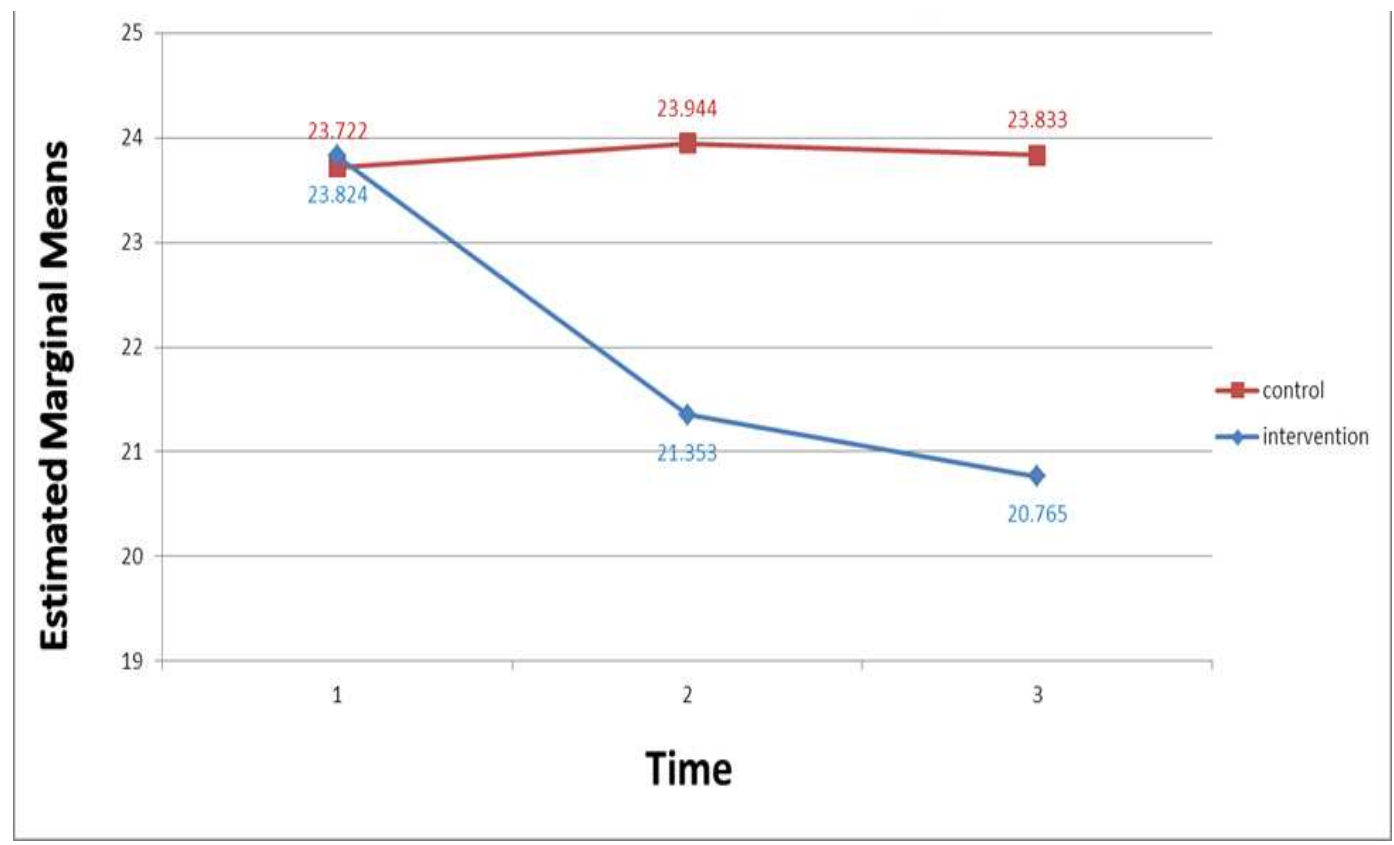

Figure 2: Estimated marginal means of extreme support

\section{DISCUSSION}

This study aimed to determine the effect of parental management education programs on the parent/child relationship in the parents and children with ADHD in Saveh city. The results of this study with determining the parental/infant parenting ratio of children with ADHD before 
intervention showed that the mean score and standard deviation of the parent $\sim$ child relationship in the intervention group before the intervention in the care and extreme support indexes were $16.7 \pm 5.8$ and $24.4 \pm 7.4$, respectively, and in the control group in the care and extreme support indexes were $18.5 \pm 6.6$ and $4.20 \pm 0.5$, respectively which are in line with Khanjani et al. (2014) study. This study showed that a good relationship between parents and their children was associated with high levels of self esteem and mental health. However, the separation of parents and having unfavorable relationships with children were discontinuous and negative with low levels of self $\sim$ esteem, depression, and hostile behaviors. In a study conducted by Esmaeilinasab 2009, Parent Management Education Program could have a positive effect on parenting skills, better child management, and reduction of ADHD symptoms.

Regarding the determination of parental/infant relationship immediately and one month after the training program for parents, it was found that immediately after training, the mean and standard deviation of the parent $\sim$ child relationship score was $18.6 \pm 6.5$ and the protection index was higher in the group Control $\pm 1.24 \pm 0.5$, and the mean and standard deviation of the care and the extreme support indexes were $22.1 \pm 2.5$ and $20.8 \pm 4.4$, respectively, and one month after the training in the control group the mean and standard deviation of the parent child care and extreme support indexes were $18.7 \pm 6.4$ and $23.8 \pm 5.2$, respectively, and in the intervention group, the mean and standard deviation of the care and extreme support indexes were $26.7 \pm 4.6$ and $20.6 \pm 2.2$, respectively. This suggests that the parental management training program improves the parent $\sim$ child relationship in the parents of children with ADHD. The results of the Tiano, 2011 and Mcneil study, was consistent with the results of this study, suggesting that the program of parental management can promote parent child relationships in the parents of children with ADHD. In the study of Tahmatsian et al. (2011), the results showed that behavioral training program is effective in improving the parent-child function, reducing the parents' anxiety and stress, and increasing their self - esteem. The results of the study of Danforth et al (2010) also coincide with the results of this study. Besides, the results of this study concerning the comparison of parent $\sim$ child relationship showed that before, immediately, and one month after the implementation of the parent management training program, the ratio of the parent $\sim$ child relationship after the intervention was upward, so that the highest score, the parent -child relationship was one month after the parent management training program, which is in line with the results of the Rossiter and Lavaque study, 1995 and Mccart and Priester, 2011. Also, this finding is in line with the results of Kurdestani et al. (2014) and Fermand (2011) studies. The results of this study were confirmed by the results of the majority of the mentioned studies. The results of this study showed that the parent education program improves the parent $\sim$ child relationship in parents of children with (ADHD) and also, the parent management training program is necessary.

\section{CONCLUSION}

The results of this study showed that parenting education program has a positive effect on the parent $\sim$ child relationship in the children with ADHD and it is significant in $\mathrm{P} \leq 0.05$. Since the parents of children with ADHD usually use negative parenting practices and give less self $\sim$ esteem in child -rearing, learning more effective management and parenting practices can help parents to adopt their children. Making a parental management training as a therapeutic option along 
with treatments also provides the mental health and comfort of the family, which results in parents having a positive attitude and a better sense of life more responsibly and, in the end, they will deal with their children's problems more readily. Parental management education is of paramount importance and can increase parent child relationship and adaptability, relaxation, reduction of empathy distress, and quality of life in families with ADHD children.

\section{References}

Barkley, R. A., Shelton, T. L., Crosswait, C., Moorehouse, M., Fletcher, K., Barrett, S \& Metevia, L. (2000). Multi method psycho educational intervention for preschool children with disruptive behavior: Preliminary results at post-treatment. The Journal of Child Psychology and Psychiatry and Allied Disciplines, 41(3), 319 332.

Danforth, J. S. (2010). Training parents of children with comorbid attention deficit hyperactivity disorder and oppositional defiant disorder. Handbook of parent training: helping parents prevent and solve problem behaviors, 3, 345 378.

Enebrink, P., Högström, J., Forster, M., \& Ghaderi, A. (2012). Internet based parent management training: A randomized controlled study. Behavior research and therapy, 50(4), 240 249.

Esmaeilinasab M, Alizadeh H, Ahadi H, \& Delevan As. (2009) Comparing The Effectiveness Of Behavioral Parent Training With The Approach Of Adler And Reduce The Severity Of Symptoms Of Conduct Disorder In Children. Journal of Exceptional Children. 37(10): 227 236 .

Hetchman, L. (2009). Attention - deficit disorders. In: Sadock, BJ, Sadock, VA. Eds, Kaplan \& Sadock Comprehensive Textbook of Psychiatry. 8th ed. Vol. 2. Baltimor: Lippincott Wiliams \& Wilkins: 3183.

KHanjani Z, Amini S, Malek As, \& Hashemi T. (2014). Parent Management Training To Improve Attention Deficit-Hyperactivity Syndrome in Children. Yolutionary Psychology: Psychologists Iran. 10 (39), 321 311.

KHoshabi K, Moradi SH, \& SHojaee S. (2013). Prevalence of Behavioral Disorders among Primary School Students in Ilam Province. Rehabilitation in Diseases and Mental Disorders. $8(29): 33 \sim 28$.

McCart, M. R., Priester, P. E., Davies, W. H., \& Azen, R. (2006). Differential effectiveness of behavioral parent training and cognitive behavioral therapy for antisocial youth: A meta analysis. Journal of abnormal child psychology, 34(4), 525 541.

Östberg, M., \& Rydell, A. M. (2012). An efficacy study of a combined parent and teacher management training program for children with ADHD. Nordic journal of psychiatry, $66(2), 123 \sim 130$. 
Parker, G., Tupling, H., \& Brown, L. B. (1979). A parental bonding instrument. British journal of medical psychology, 52(1), 1 10 .

Pelham, W. Hoza, B. (2010). Intensive Treatment: A Summer Treatment Program for Children with Adhd, Psychosocial Treatments for Child and Adolescent Disorders. Empirically Based Strategies for Clinical Practice, 311 340.

Rajablo, M. (2012). The Effectiveness of Management Education to Parents on Reducing Symptoms of Attention Deficit / Excessive Kinetic Disorders. 11 25. M.Sc. Thesis, Allameh Tabataba'i University.

Rossiter, D. T. R., \& La Vaque, T. J. (1995). A comparison of EEG biofeedback and psychostimulants in treating attention deficit/hyperactivity disorders. Journal of Neurotherapy, 1(1), 48 59.

Sadock B, Sadock V, \& Rezaee Translation. (2014). Summary of Psychiatry and Behavioral Sciences Clinical Psychiatry Kaplan Sadock. Tehran, Publishers: Arjmand. 